\title{
Laparoscopic proximal gastrectomy with a hand-sewn esophago-gastric anastomosis using a knifeless endoscopic linear stapler
}

\author{
Hiroshi Okabe $\cdot$ Kazutaka Obama • \\ Eiji Tanaka $\cdot$ Shigeru Tsunoda $\cdot$ Masatoshi Akagami $\cdot$ \\ Yoshiharu Sakai
}

Received: 9 March 2012/ Accepted: 5 July 2012/Published online: 24 July 2012

(C) The International Gastric Cancer Association and The Japanese Gastric Cancer Association 2012

\begin{abstract}
Proximal gastrectomy has been applied for selected patients with early upper gastric cancer, because of its potential advantages over total gastrectomy, such as preserving gastric capacity and entailing fewer hormonal and nutritional deficiencies. Esophago-gastric anastomosis is a simple reconstruction method with an excellent postoperative outcome provided that gastroesophageal reflux is properly prevented. Following open surgery, the esophagus is anastomosed to the anterior stomach wall with partial fundoplication to prevent esophageal reflux. We developed a novel laparoscopic hand-sewn method to reproduce the anti-reflux procedure that is used in open surgery. The esophagus is first fixed to the anterior stomach wall with a knifeless endoscopic linear stapler. This fixation contributes to maintaining a stable field for easier hand-sewn anastomosis, and allows us to complete the left side of the fundoplication at the same time. This novel technique was used to successfully perform complete laparoscopic proximal gastrectomy with a hand-sewn esophago-gastric anastomosis in ten patients, without any postoperative complications. No patient had symptoms of gastroesophageal reflux during a median follow-up period of 19.9 months. One patient developed anastomotic stenosis, and this was resolved with endoscopic dilatation. The mean percent body weight loss at 12 months after surgery, in comparison to the preoperative weight, was $10.4 \%$. Laparoscopic proximal gastrectomy with an esophago-gastric anastomosis using our novel technique would be a feasible
\end{abstract}

\footnotetext{
H. Okabe $(\square) \cdot$ K. Obama $\cdot$ E. Tanaka $\cdot$ S. Tsunoda

M. Akagami · Y. Sakai

Department of Surgery, Graduate School of Medicine

Kyoto University, 54 Kawahara-cho, Shogoin, Sakyo-ku,

Kyoto 606-8507, Japan

e-mail: hokabe@kuhp.kyoto-u.ac.jp
}

choice would be a feasible choice and would show benefit for selected patients with early upper gastric cancer.

Keywords Laparoscopic surgery - Gastric cancer . Esophago-gastric anastomosis · Proximal gastrectomy

\section{Introduction}

Gastric cancer is one of the most common cancers worldwide. Many patients in Eastern Asia, where gastric cancer is one of the main causes of cancer death, are diagnosed with early disease. Although total gastrectomy is considered to be the standard operation for an upper gastric cancer, proximal gastrectomy has been applied for selected patients with early gastric cancer as a less invasive and function-preserving surgery. Proximal gastrectomy has potential advantages over total gastrectomy, such as preserving gastric capacity and entailing fewer hormonal and nutritional deficiencies [1]. Following proximal gastrectomy, reconstruction is done with either an esophago-gastrostomy or jejunal interposition. The esophago-gastric anastomosis is a simpler procedure, resulting in a shorter operation time. A retrospective survey using a questionnaire has shown that esophago-gastric anastomosis has a better outcome, with regard to abdominal symptoms, than jejunal interposition [2]. However, a higher incidence of postoperative gastroesophageal regurgitation has also been reported with the esophago-gastric anastomosis, and this prevents its widespread use [3]. Although jejunal interposition is favored by some surgeons in this respect, the procedure is technically demanding and requires a longer operation time [4]. Furthermore, late complications, such as jejunitis, stasis, and ileus, have been reported. Difficulty in endoscopic surveillance following jejunal interposition 
has also been reported [5]. Given the findings for these two methods, there seems to be a need to establish an optimal reconstruction method after proximal gastrectomy.

Several techniques of esophago-gastric anastomosis to prevent esophageal reflux have been reported. The esophagus is recommended to be anastomosed to the anterior wall in the center of the stomach [6]. This results in the remnant stomach surrounding the dorsal half of the lower esophagus, increasing the pressure at the lower end of the esophagus and thereby preventing esophageal reflux by mechanisms similar to those of partial fundoplication. Although a gastroesophageal anastomosis can be done with a circular stapler, anastomotic stenosis can occur in 6-20\% of patients a few months or even later after such a procedure $[7,8]$. We have adopted a hand-sewn anterior esophago-gastric anastomosis in open proximal gastrectomy because the incidence of stenosis was reported to be lower with hand-sewn sutures [9].

Laparoscopic surgery has been accepted as a standard option for early gastric cancer, especially in Japan and Korea. Laparoscopic proximal gastrectomy with esophagogastrostomy would be an ideal minimally invasive approach for early upper gastric cancer, if gastroesophageal reflux could be properly prevented. Laparoscopic esophago-gastrostomy using a linear stapler was first reported by Uyama et al. [10]. However, reflux esophagitis was still observed in nearly half of the patients [11].

Here we report our novel laparoscopic procedure using a knifeless endoscopic linear stapler for the easier creation of a hand-sewn anterior esophago-gastric anastomosis, which resulted in excellent postoperative function.

\section{Patients and methods}

\section{Patients}

Ten patients ( 6 males and 4 females) underwent laparoscopic proximal gastrectomy at Kyoto University Hospital between August 2009 and December 2011. These patients had a median age of 65 years (range 31-83 years) and a body mass index of 22.9 (range 18.3-27.1). Nine patients were diagnosed with gastric cancer in the upper third of the stomach without lymph node involvement, and one patient was diagnosed with a gastrointestinal stromal tumor. Although early cancer is the recommended indication for proximal gastrectomy according to the Japanese gastric cancer treatment guidelines, in this study two patients with small $\mathrm{T} 2$ tumors that were beyond the indication were included [12]. The diagnosis was based on preoperative examinations including gastrointestinal endoscopy, an upper gastrointestinal series, and abdominal computed tomography. The 3rd English edition of the Japanese classification of gastric carcinoma was used [13].

\section{Surgical technique}

Proximal gastrectomy

The patient is placed in a modified lithotomy position. The first port is placed through the umbilicus using an open method. The laparoscope is inserted via the umbilical port, and four operating ports and a Nathanson liver retractor are placed as indicated in Fig. 1. The surgeon stands on the patient's right side. The extent of lymph node dissection is basically $\mathrm{D} 1+$, according to the Japanese gastric cancer treatment guidelines 2010 (version 3) [12]. The left side of the greater omentum is first separated and the left gastroepiploic vessels are divided to dissect lymph node (LN) station \#4sb. The gastrosplenic ligament is separated, and the short gastric vessels are divided to harvest $L N$ station \#4sa. The lesser omentum is opened to enter the lesser sac. The perigastric vessels along the lesser curvature are cut at the point between the right and left gastric arterial arcade. The upper half of the lesser curvature is skeletonized toward the proximal side to remove LN station \#3a. The exact location of the tumor is determined by intraoperative endoscopy and the stomach is transected using an endoscopic linear stapler to secure the distal margin.

LN station \#8a is dissected along the common hepatic artery, and LN stations \#7, 9, and $11 \mathrm{p}$ are removed en bloc by a medial approach, which has been described elsewhere [14]. The gastrophrenic fold is divided along the right crus of the diaphragm towards the esophagus and the fundic portion of the stomach is fully mobilized from the retroperitoneum. The posterior vagal trunk is identified at the posterior side of the esophago-gastric junction. The celiac

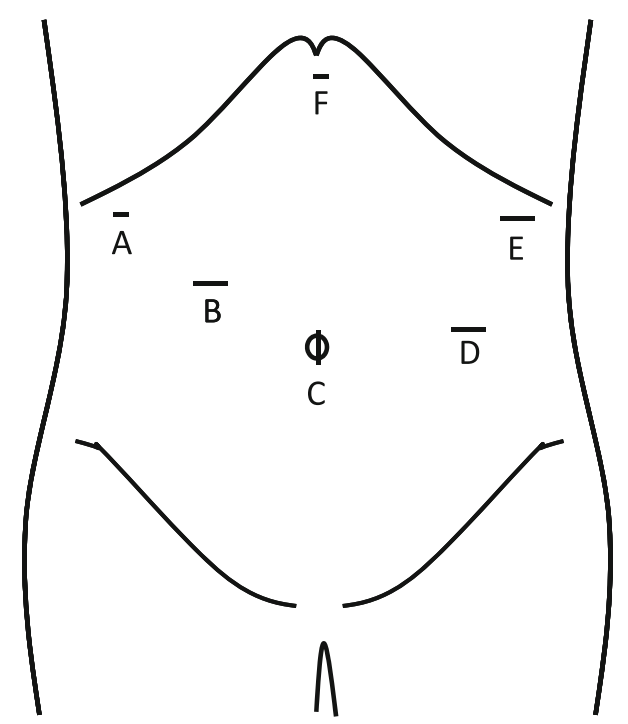

Fig. 1 Port sites for laparoscopic proximal gastrectomy. A $5 \mathrm{~mm}$ port, $B 12 \mathrm{~mm}$ port, $C$ laparoscope through the umbilicus, $D 12 \mathrm{~mm}$ port, $E 12 \mathrm{~mm}$ port, $F$ Nathanson liver retractor 
Fig. 2 Schematic outline of the reconstruction with an esophago-gastrostomy using a knifeless linear stapler and hand suturing. a After a $3 \mathrm{~cm}$ incision is made on the anterior wall of the remnant stomach, a knifeless endoscopic linear stapler is applied between the stomach and the left side of the esophagus. A naso-gastric tube is used as a guide. $\mathbf{b}$ The left side of the esophagus is fixed to the stomach wall. The stump of the esophagus is opened and adjusted to the size of the previous $3 \mathrm{~cm}$ incision on the stomach. Part of the right edge of the esophagus is retained in most cases. c Posterior running sutures are performed. d The anastomosis is completed with an anterior running suture. The gastric stump is anchored to the right crus of the diaphragm (white arrow). The posterior partial fundoplication is done with interrupted sutures (black arrows)

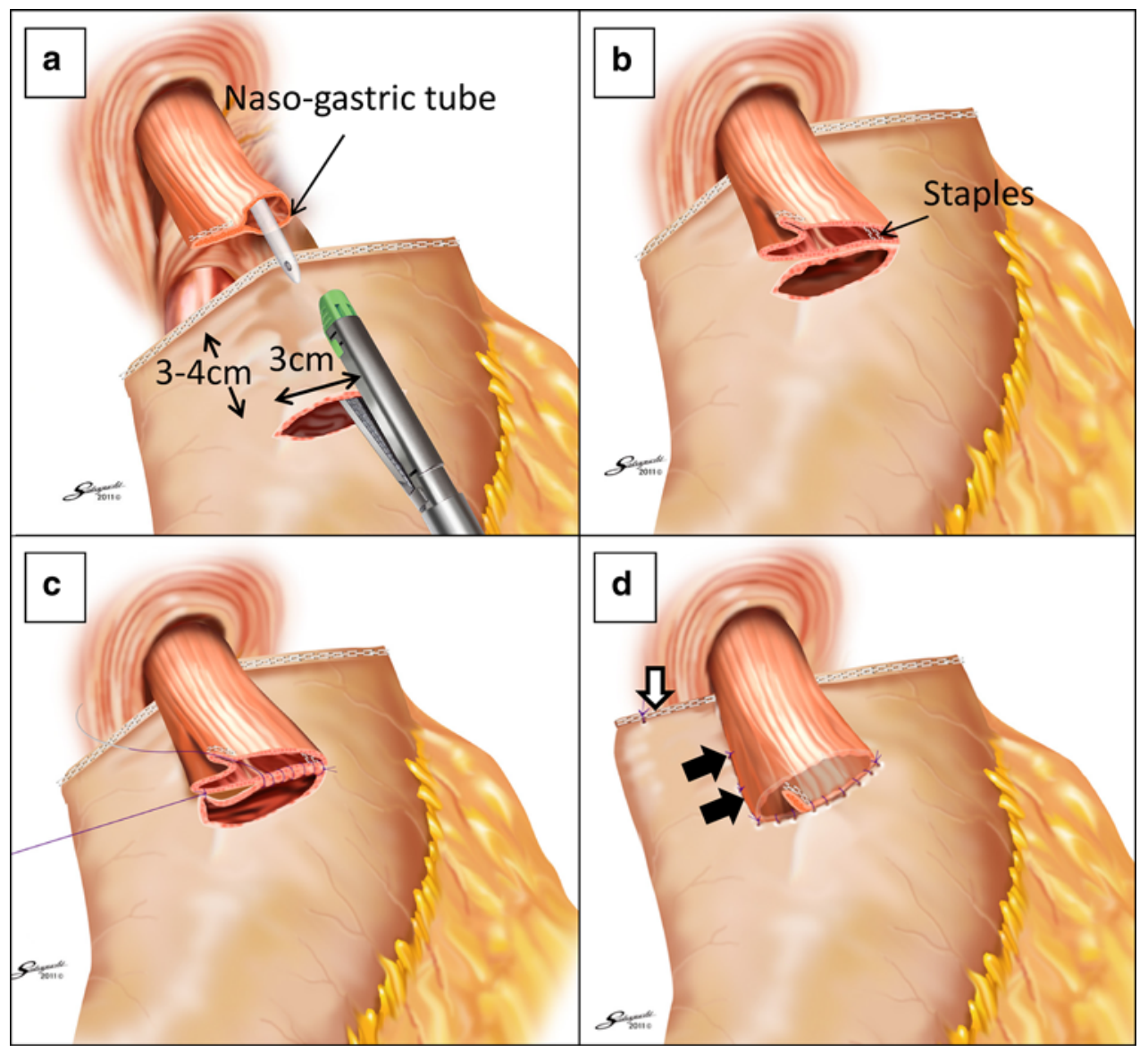

branch of the vagal trunk is preserved, while the left gastric artery is divided to remove LN station \#7. LN station \#9 is dissected and separated from the crus of the diaphragm. The esophagus is completely isolated and transected with an endoscopic linear stapler. Finally, the gastric fundus is retracted caudally by the assistant, and LN station \#11 is dissected along the splenic artery in a distal direction to complete the proximal gastrectomy. The specimen is removed through the extension of the umbilical port wound.

\section{Esophago-gastrostomy}

The center of the anterior wall of the remnant stomach is transversely opened for $3 \mathrm{~cm}$, approximately $3-4 \mathrm{~cm}$ from the edge (Fig. 2a). The gastric juice is suctioned thorough the opening to prevent any contamination. The left border of the esophageal edge is incised, and a naso-gastric tube is advanced through the opening to confirm the "true" esophageal lumen. The anvil side of a knifeless endoscopic stapler (ENDOPATH ${ }^{\circledR}$ ETS $45 \mathrm{~mm}$ No Knife, Ethicon Endo-Surgery, Cincinnati, $\mathrm{OH}$ ) is inserted into the hole in the remnant stomach. The cartridge side of the stapler is inserted into the esophagus using the naso-gastric tube as a guide. The knifeless stapler is used to fix the left side of the esophagus to the anterior stomach wall (Figs. 2b, 3a). The esophageal edge is opened and adjusted to the size of the previous $3-\mathrm{cm}$ incision on the stomach. Part of the right edge of the esophagus is retained in most cases (Fig. 2b). A posterior wall anastomosis is performed by continuous hand suturing, using 3-0 Vicryl ${ }^{\circledR}$, Ethicon Endo-Surgery, Cincinnati, $\mathrm{OH}$ (Figs. 2c, 3b, c). The anterior wall is also closed with continuous sutures (Fig. 3d). After the anastomosis has been examined for completeness, a few reinforcement sutures are placed if required. The right-side stump of the remnant stomach is anchored to the right crus of the diaphragm to prevent dislocation of the stomach (Figs. 2d, 4a). Finally, the right side of the lower esophagus is fixed to the stomach wall to complete a posterior 180-degree fundoplication (Figs. 2d, 4b).

\section{Results}

The patients' backgrounds and surgical outcomes are listed in Table 1. All patients had tumors in the upper third of the stomach; nine patients had gastric cancer and one had a gastrointestinal stromal tumor (GIST). The seven patients 

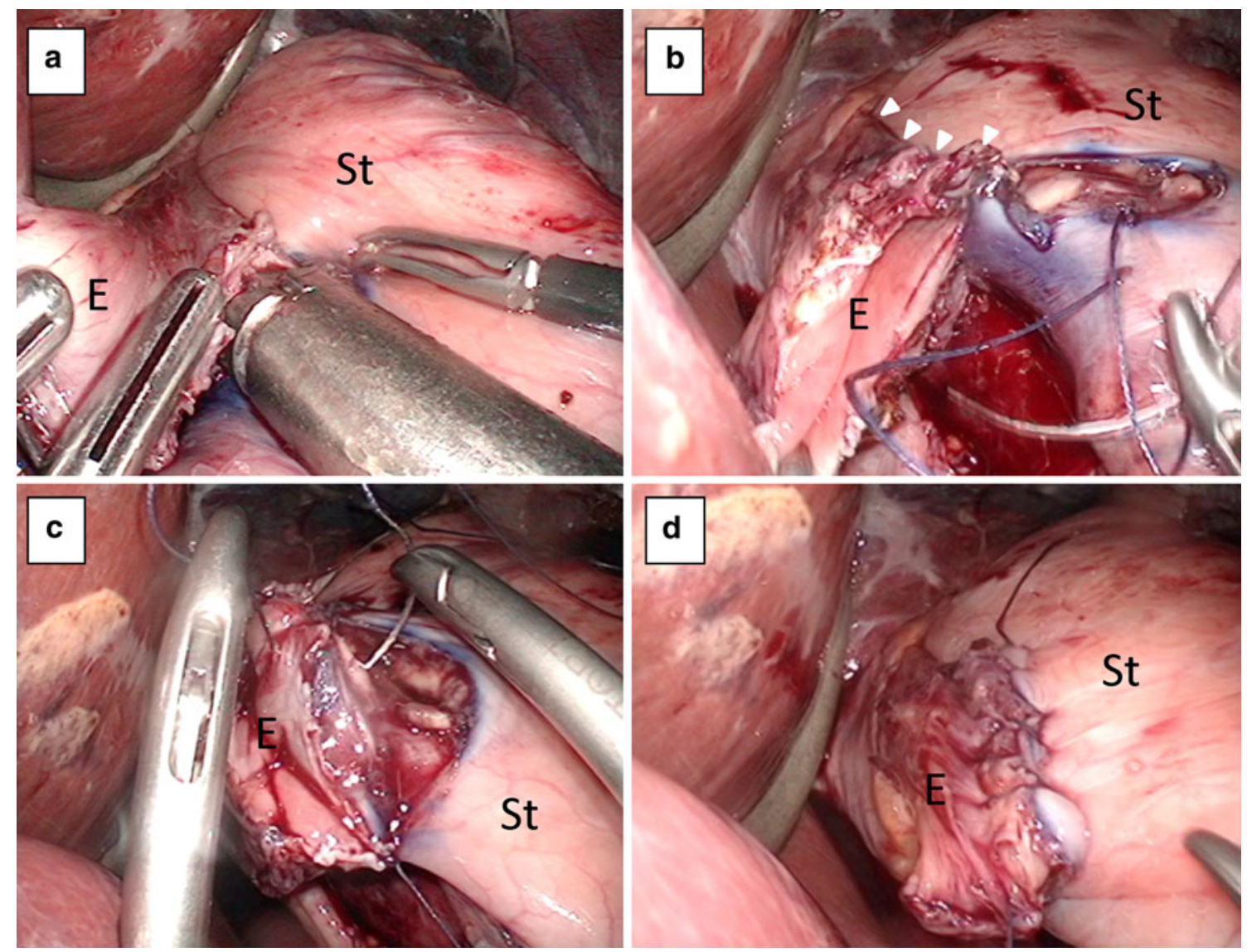

Fig. 3 Intraoperative view during the esophago-gastric anastomosis. a Application of knifeless endoscopic linear stapler. b A stay suture between the right edge of the esophageal opening and the hole on the

fixed to the stomach wall in the previous step (white arrowheads). c A posterior running suture is done from the left to the right edge. d Completion of the anastomosis with an anterior running suture. $E$ esophagus, $S t$ the remnant stomach

with a clinical $\mathrm{T} 1 \mathrm{~b}$ tumor underwent $\mathrm{D} 1+$ lymph node dissection according to the Japanese gastric cancer treatment guidelines (version 3). In the two patients with clinical T2 tumors, lymph node station \#11d was also removed. The patient with the GIST had no lymph node dissection.

The mean operative time for the ten patients was 299 min (range 174-394 min), and the estimated blood loss was $65 \mathrm{~g}$ (range $0-325 \mathrm{~g}$ ). The mean duration for the reconstruction was $82 \mathrm{~min}$. There was no conversion to open surgery. All patients recovered uneventfully without any complications and were discharged between 8 and 18 days after the surgery; the mean length of the hospital stay was 15 days. However, pathological examination revealed a positive surgical margin in one patient. Although this patient had been diagnosed with clinical T1b disease (tumor invades as far as the submucosa) before surgery, diffuse infiltrations of scattered poorly differentiated tumor cells to the serosa and to the surgical margin were revealed on pathological examination. The patient underwent laparoscopic completion gastrectomy on the 21 st day after the initial surgery to achieve an R0 resection.

The median follow-up period for the other nine patients was 21.5 months (range 5.6-32.4 months). One patient had an anastomotic stenosis at 4 months after the surgery, and this was resolved by serial endoscopic dilatation of the anastomotic site. No patient exhibited symptoms suggestive of acid reflux, such as heartburn, chest pain, or chronic cough. A proton pump inhibitor was administered for the first few months following surgery and then discontinued after the confirmation of no endoscopic finding of reflux esophagitis. Eight patients had an endoscopic follow up at least once after the surgery. Grade B esophagitis according to the Los Angeles classification was found in one patient 18 months after the surgery, although she had no symptoms of reflux. Postoperative endoscopic findings in a representative case are shown in Fig. 5. The mean percent body weight loss in comparison to the preoperative weight in seven patients at 12 months was $10.4 \%$ (range $4.5-14.3 \%)$. 

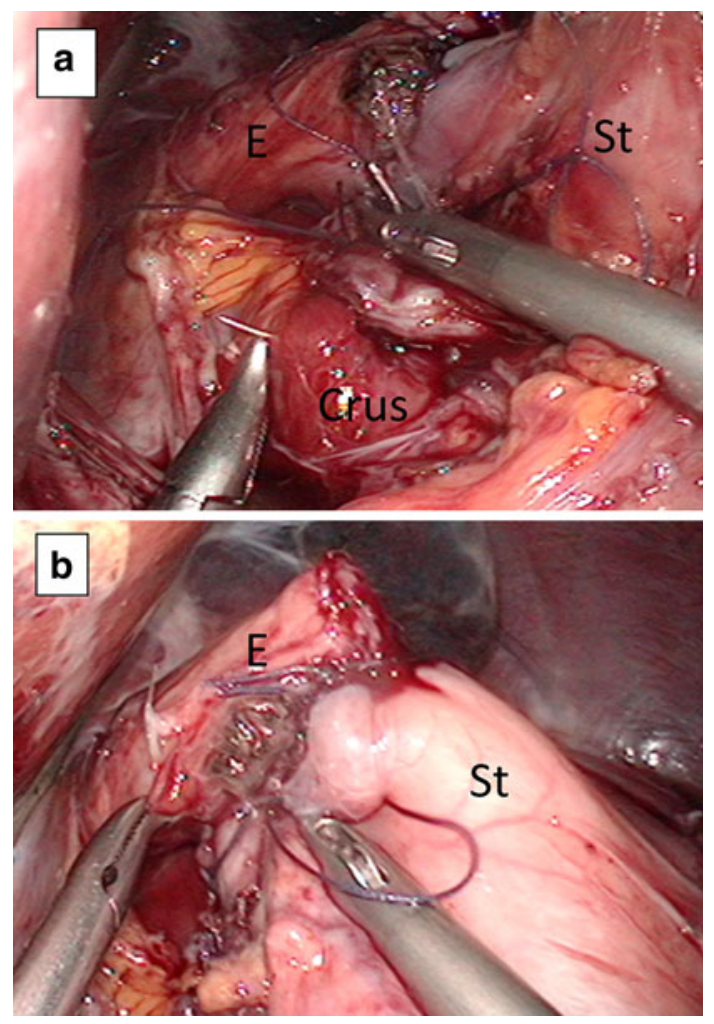

Fig. 4 Anchoring the stomach and a posterior $180^{\circ}$ fundoplication. a Anchoring the stump of the remnant stomach to the right crus of the diaphragm. b The posterior aspect of the lower esophagus is covered by the stomach wall with a few interrupted sutures. $E$ esophagus, St the remnant stomach

Table 1 Patients' backgrounds and surgical outcomes

\begin{tabular}{ll}
\hline Clinical parameters & $N=10$ \\
\hline Age (years), mean & $65(31-83)$ \\
Sex (M/F) & $6 / 4$ \\
Operative time (min), mean & $299(174-394)$ \\
Reconstruction (min), mean & $82(50-116)$ \\
Estimated blood loss (g) & $65(0-325)$ \\
Lymph node dissection (D0/D1+/D2 - 10) & a \\
Postoperative complications & $1 / 7 / 2$ \\
Mortality & $0(0 \%)$ \\
Length of postoperative hospital stay (days) & $0(0 \%)$ \\
\hline
\end{tabular}

${ }^{a}$ D0 dissection was done for one patient who had a gastrointestinal stromal tumor (GIST)

\section{Discussion}

Although there is no high-level evidence of oncological safety based on large-scale randomized controlled trials, laparoscopic surgery for early gastric cancer has been widely accepted in Eastern Asian countries. An early gastric cancer lesion limited to the upper stomach is considered as a possible indication for proximal gastrectomy in open surgery. In patients with small lesions, proximal gastrectomy with esophago-gastrostomy has several advantages in comparison to total gastrectomy, such as a shorter operative time, less postoperative body weight loss, and fewer nutritional deficiencies [1]. Therefore, establishing a laparoscopic procedure for proximal gastrectomy with esophago-gastrostomy would be an ideal approach for patients with small early upper gastric cancer.

The major reason preventing the widespread introduction of laparoscopic proximal gastrectomy with esophagogastric anastomosis is the lack of a secure method for laparoscopic anastomosis with an anti-reflux mechanism. In open surgery, the anastomosis is made at the anterior wall of the stomach and the dorsal side of the esophagus is covered with the remnant stomach, in the same manner as in partial fundoplication [6, 15]. Gastro-esophageal reflux is thereby prevented by the increased pressure at the lower end of the esophagus. In previous reports of laparoscopic esophago-gastrostomy, the anastomosis has been made with a linear stapler, with hand-sewn closure of the entry hole $[10,11]$. Side-to-side anastomosis between the anterior gastric wall and the dorsal side of the esophagus diminishes the anti-reflux high-pressure zone at the lower end of the esophagus.

We introduced laparoscopic hand-sewn anastomosis in order to keep the lower esophagus intact, and to reproduce the open surgery anti-reflux procedure under laparoscopy. We first tried a "purely" hand-sewn anastomosis. However, it was technically highly demanding because the esophageal stump was retracted into the mediastinum after transection and there was a strong tension between the esophageal end and the anterior gastric wall. In our new method, we first fixed the esophagus to the gastric wall using a knifeless linear stapler. This technique allowed easier manipulation of the esophageal stump and suturing for the anastomosis. This fixation also contributes to the mechanism of reflux prevention by completion of the left side of the fundoplication at the same time as the anastomosis is created. An anastomosis with a similar shape may also be created with a circular stapler. However, it would be more difficult to manipulate a circular stapler in a limited space than a linear stapler. In addition, the use of a circular stapler could result in a higher rate of anastomotic stenosis (which is the most frequent complication following proximal gastrectomy), in comparison to the rate with a hand-sewn anastomosis [7, 8]. In the present study, the mean duration for the reconstruction with the hand-sewn anastomosis was about $80 \mathrm{~min}$. However, the time required for reconstruction was gradually reduced to about $50 \mathrm{~min}$ by the tenth case, and it will get shorter as we gain more experience with the laparoscopic hand-sewn technique. 
Fig. 5 a, b Gastrographin study in a representative case on the 7 th postoperative day. a Smooth passage of gastrographin through the anastomosis. b No reflux is seen in the head-down position. c, d Endoscopic views of the anastomosis at 6 months after surgery. $\mathbf{c}$ No finding of gastroesophageal reflux at the lower esophagus. The staple line is not identified. $\mathbf{d}$ A fundus-like space is seen behind the new esophagogastric junction
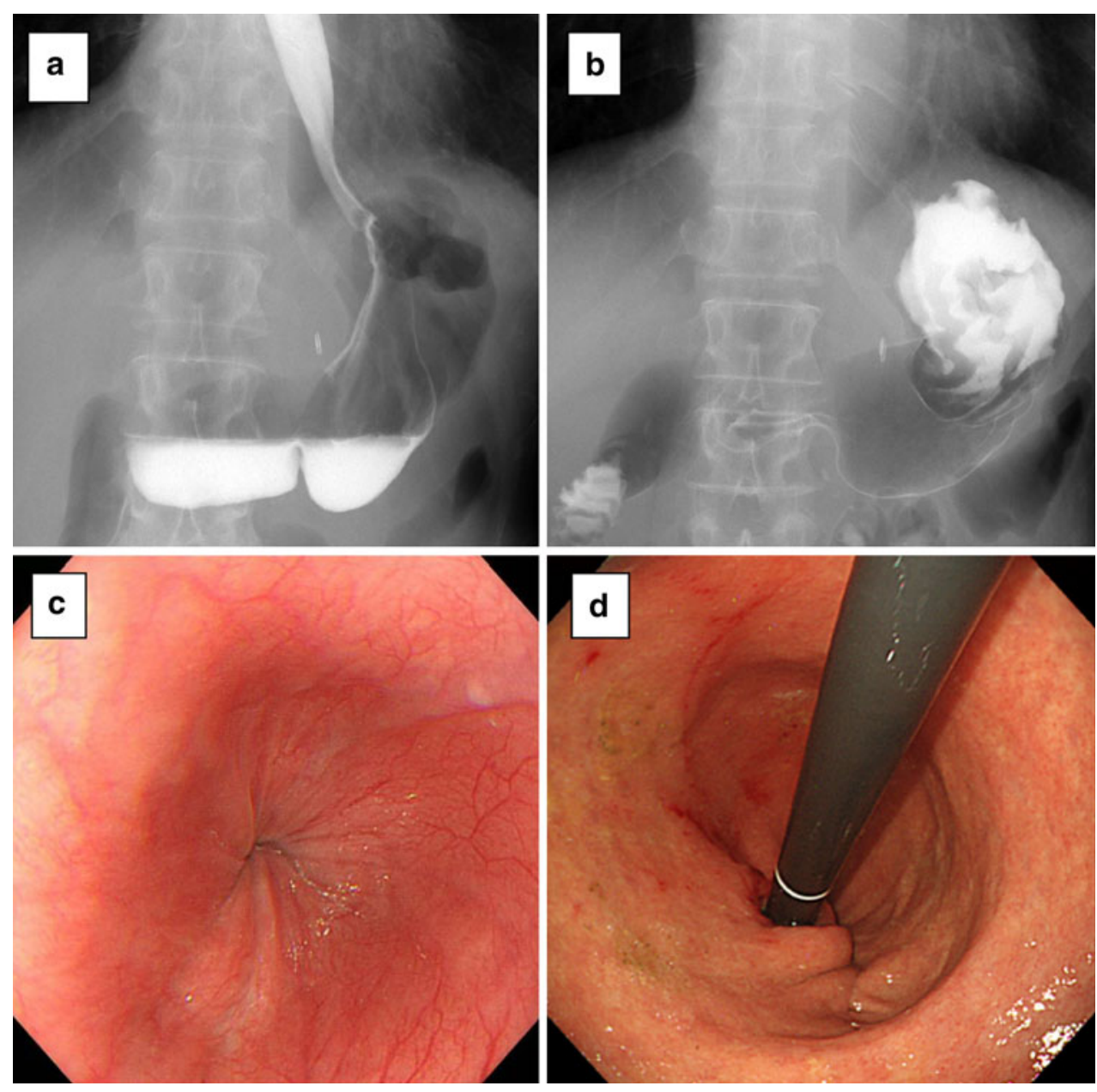

Preserving the abdominal esophagus and a larger stomach is important to maintain the capacity of the remnant stomach and to form an effective anti-reflux anastomosis. Therefore, the point of gastric transection should be determined precisely. We examined the lateral extension of cancer by preoperative endoscopy and placed clips at appropriate points with a negative biopsy. The resection margin was determined to include these clips using intraoperative endoscopy. However, the distal resection margin was positive in one patient. The tumor in this patient had been preoperatively diagnosed as T1b. However, the pathological examination revealed that scattered cancer cells had infiltrated into the muscular and the subserosal layer, without mucosal involvement. Confirmation of a negative surgical margin by examination of a frozen section during the operation is recommended when proximal gastrectomy is applied for this histological type of lesion.

Although there were no postoperative complications, the mean length of postoperative hospital stay in the present study seemed to be a little longer than expected. One possible reason for this is that we did not apply a clinical pathway for patients with proximal gastrectomy. Some patients requested a longer stay in the hospital. No patient had reflux symptoms during the follow-up period, although endoscopic examination revealed Grade B esophagitis in one patient. Another patient had mild symptoms of stenosis, and the stenosis was easily resolved with endoscopic balloon dilatations. The length of the incision on the stomach is important for avoiding stenosis, because it determines the size of the stoma. The mean percent body weight loss in our patients at 12 months after surgery was $10 \%$, which seems to be less than that with total gastrectomy $[16,17]$. Although the nutritional status was not examined in detail in this study, proximal gastrectomy may also benefit patients from the nutritional point of view. Careful and longer follow up of more patients is necessary to confirm these observations.

In summary, the short-term outcome of this novel technique of laparoscopic esophago-gastrostomy was excellent. Fixation of the esophagus to the anterior stomach wall with a knifeless linear stapler contributes to an easier hand-sewn anastomosis and to completion of the fundoplication. We believe our method is a feasible choice for selected patients. However, careful and longer follow up of 
more patients is necessary to determine the advantages of our method.

Acknowledgments We are deeply grateful to Shigeyuki Sakaguchi for the creation of the schematic illustration of a hand-sewn anastomosis.

\section{References}

1. Hinoshita E, Takahashi I, Onohara T, Nishizaki T, Matsusaka T, Wakasugi $\mathrm{K}$, et al. The nutritional advantages of proximal gastrectomy for early gastric cancer. Hepatogastroenterology. 2001;48: 1513-6.

2. Tokunaga M, Hiki N, Ohyama S, Nunobe S, Miki A, Fukunaga T, et al. Effects of reconstruction methods on a patient's quality of life after a proximal gastrectomy: subjective symptoms evaluation using questionnaire survey. Langenbecks Arch Surg. 2009;394: 637-41.

3. An JY, Youn HG, Choi MG, Noh JH, Sohn TS, Kim S. The difficult choice between total and proximal gastrectomy in proximal early gastric cancer. Am J Surg. 2008;196:587-91.

4. Katai H, Morita S, Saka M, Taniguchi H, Fukagawa T. Longterm outcome after proximal gastrectomy with jejunal interposition for suspected early cancer in the upper third of the stomach. Br J Surg. 2010;97:558-62.

5. Kikuchi S, Nemoto Y, Katada N, Sakuramoto S, Kobayashi N, Shimao H, et al. Results of follow-up endoscopy in patients who underwent proximal gastrectomy with jejunal interposition for gastric cancer. Hepatogastroenterology. 2007;54:304-7.

6. Hirai T, Matsumoto H, Iki K, Hirabayashi Y, Kawabe Y, Ikeda $\mathrm{M}$, et al. Lower esophageal sphincter- and vagus-preserving proximal partial gastrectomy for early cancer of the gastric cardia. Surg Today. 2006;36:874-8.

7. Gould J, Garren M, Boll V, Starling J. The impact of circular stapler diameter on the incidence of gastrojejunostomy stenosis and weight loss following laparoscopic Roux-en-y gastric bypass. Surg Endosc. 2006;20:1017-20.
8. Fisher B, Atkinson J, Cottam D. Incidence of gastroenterostomy stenosis in laparoscopic Roux-en-y gastric bypass using 21- or 25-mm circular stapler: a randomized prospective blinded study. Surg Obes Relat Dis. 2007;3:176-9.

9. Takeyoshi I, Ohwada S, Ogawa T, Kawashima Y, Ohya T, Kawate S, et al. Esophageal anastomosis following gastrectomy for gastric cancer: comparison of hand-sewn and stapling technique. Hepatogastroenterology. 2000;47:1026-9.

10. Uyama I, Sugioka A, Matsui H, Fujita J, Komori Y, Hatakawa Y, et al. Laparoscopic side-to-side esophagogastrostomy using a linear stapler after proximal gastrectomy. Gastric Cancer. 2001;4: 98-102.

11. Sakuramoto S, Yamashita K, Kikuchi S, Futawatari N, Katada N, Moriya $\mathrm{H}$, et al. Clinical experience of laparoscopy-assisted proximal gastrectomy with toupet-like partial fundoplication in early gastric cancer for preventing reflux esophagitis. J Am Coll Surg. 2009;209:344-51.

12. Japanese Gastric Cancer Association. Japanese gastric cancer treatment guidelines 2010 (ver. 3). Gastric Cancer. 2011;14: 113-23.

13. Japanese Gastric Cancer Association. Japanese classification of gastric carcinoma: 3rd English edition. Gastric Cancer. 2011;14: 101-12.

14. Okabe H, Obama K, Kan T, Tanaka E, Itami A, Sakai Y. Medial approach for laparoscopic total gastrectomy with splenic lymph node dissection. J Am Coll Surg. 2010;211:e1-6.

15. Ichikawa D, Komatsu S, Okamoto K, Shiozaki A, Fujiwara H. Otsuji E. Langenbecks Arch Surg: Evaluation of symptoms related to reflux esophagitis in patients with esophagogastrostomy after proximal gastrectomy; 2012 [Epub ahead of print].

16. Bae JM, Park JW, Yang HK, Kim JP. Nutritional status of gastric cancer patients after total gastrectomy. World J Surg 1998;22: 254-260; discussion 260-251.

17. Fein M, Fuchs KH, Thalheimer A, Freys SM, Heimbucher J, Thiede A. Long-term benefits of Roux-en-y pouch reconstruction after total gastrectomy: a randomized trial. Ann Surg. 2008;247: 759-65. 